\title{
A Low Power Digital Signal Processor with Adaptive Band Activation for Digital Hearing Aid Chip
}

\author{
Seung Jin Lee, Sunyoung Kim, and Hoi-Jun Yoo \\ Department of EECS \\ Korea Advanced Institute of Science and Technology (KAIST) \\ Daejeon, Republic of Korea \\ seungjin@eeinfo.kaist.ac.kr
}

\begin{abstract}
A low power digital signal processor (DSP) for a digital hearing aid chip is presented. The DSP integrates three programmable digital finite impulse response (FIR) filters. Each FIR filter can have one pass-frequency out of seven preset frequencies so that only three FIR filters can have the same flexibility as seven filters. Additionally, a silence mode is defined in which only one filter is activated. A digital voice activity detection circuit is implemented for this purpose. The DSP is implemented as part of a fully integrated digital hearing aid chip. It uses a $0.18 \mu \mathrm{m}$ CMOS process and occupies an area of $0.5 \mathrm{~mm}^{2}$. Power consumption is $25 \mu \mathrm{W}$ in normal operating mode and $9 \mu \mathrm{W}$ in silence mode at $0.9-\mathrm{V}$ supply.
\end{abstract}

\section{INTRODUCTION}

Two of the major issues in digital hearing aid design are high flexibility and low power consumption. High flexibility accounts for a more accurate fitting that results in increased hearing clarity for the wearer. Lower power consumption prolongs battery lifetime. There have been active researches to achieve both high flexibility and low power consumption at once. One approach is to use a single configurable filter to divide only hi and lo bands [1] [2]. While this approach is simple and power efficient, it is only applicable to patients with specifically shaped audiograms and thus has limited flexibility. A more elaborate approach is to divide the frequency spectrum into many bands [3] [4]. Recent works have used up to 16 bands. While these implementations do allow for a fine-grained fitting, there is a large power penalty for having such a large number of filters. In addition, the high flexibility is seldom fully appreciated because audiograms of typical hearing impaired patients are not very complex [5].

In this paper, we propose a digital signal processor (DSP) that has three finite impulse response (FIR) filters. It has the low power consumption of three FIR filters and the high flexibility of seven frequency bands. Each filter can be configured to pass one of seven different frequency bands. In designing the filters, instead of using the general purpose DSP, a hardwired digital FIR filter with programmable parameters is used. Filter coefficients for all seven frequency bands are found using powers of 2 so that multiplications can be implemented using simple shift operations.

The main purpose for accurate fitting of hearing aids is to enhance the patients' ability to comprehend spoken language. Most other sounds such as environmental noise can be perceived with a far less accurate compensation. This presents an opportunity to decrease power consumption by reducing the complexity of the DSP when the patient is not listening to speech. By using a voice activity detection circuit with low computational complexity in conjunction with clock gating techniques, the number of active filter elements can be reduced further to just one when no speech is detected in the incoming sound stream.

\section{SYSTEM OVERVIEW}

Fig. 1 shows the overall architecture of the proposed DSP. The input signal is first examined by the voice activity detection circuitry. Depending on the contents of the sound signal, the DSP operates in one of two modes; active mode and silence mode. When speech is detected, the DSP runs in the active mode, in which all three filters of the FIR filter bank are fully activated. Each filter is a flexible dedicated FIR circuit that can be configured to have a pass-frequency among $0,2,3,4,6,7$, and $8 \mathrm{kHz}$. The outputs of these filters are combined by the mixer to obtain the desired frequency dependent gain compensation. The silence mode of the DSP is enabled after speech is not detected. In this mode, since

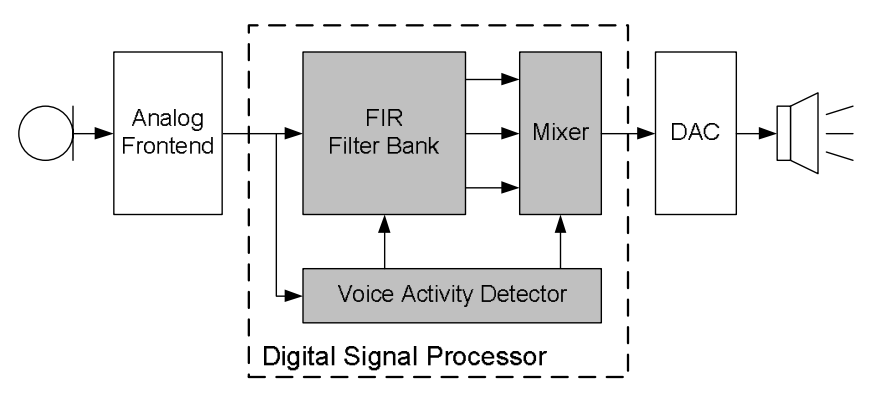

Figure 1. Proposed digital signal processor. 
the need for accurate compensation is diminished, only one of the filters in the filter bank is activated by gating off the clock signal to two of the filters. The parameters for the active filter are dynamically adjusted to make up for the absence of signals from the other filters. In order to ensure a smooth transition between the normal operating mode and the power saving mode, the mixer includes a fade-in/fade-out function.

\section{SySTEM COMPONENTS}

\section{A. Filter Bank}

Splitting the frequency spectrum into as many miniscule bands as possible and assigning appropriate gains to each band would result in a better fit. Recently up to sixteen filters [3] have been used in an effort to achieve a high level of flexibility. Increasing the number of filters enhances the likelihood for a better fitting, but such an implementation would not only require a large amount of processing power, since the audiograms of most patients suffering from hearing loss have nearly monotonic curves [5], a lot of power would be wasted. Using a custom fitting algorithm in conjunction with our proposed design, we have found that three configurable filters are sufficient for achieving the desired frequency gain compensation for typical cases of hearing impairment [6].

Fig. 2 shows the proposed digital filter bank. Three configurable low power FIR filters are used to cover three bands. Dedicated hardware filters are chosen over the general purpose DSP due to their low power and small size characteristics, and FIR filters are chosen over infinite impulse response (IIR) filters to avoid the IIR filter's overflow and limit cycles problems [7]. Fig. 3 is a frequency gain plot of the filters configured for each of the possible preset frequencies with typical values for other parameters. The center frequencies of the bands are concentrated around the frequency range between 2 to $8 \mathrm{kHz}$ since that is where most hearing loss actually occurs. It can be seen that the bands overlap by a fairly large amount: up to $60 \%$ of the pass-band. Although this kind of overlapping may be undesirable in the traditional multi-band approach, it is required to obtain a smooth frequency gain compensation graph by using just three bands.

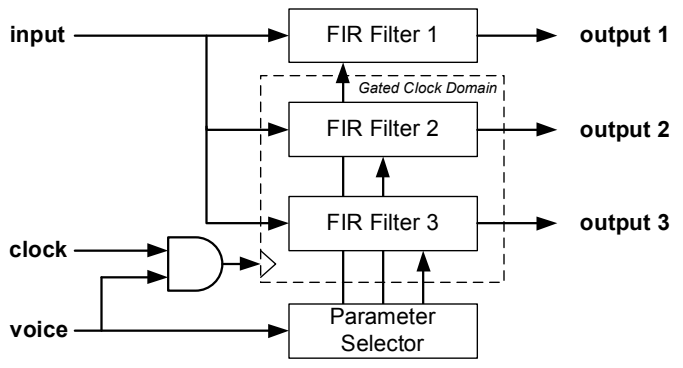

Figure 2. Digital filter bank.

\section{B. Finite Impulse Response Filter}

The FIR filter structure is shown in Fig. 4. It is implemented using a band-pass and notch filter [8]. This approach gives results that are similar to low-pass/high-pass structures while maintaining lower power dissipation and smaller size. Each FIR sub-filter outputs a band-pass component with a transfer function given by

$$
H_{\text {band-pass }}(z)=0.5+a_{0} z^{-2}+a_{1} z^{-4}+a_{2} z^{-6}+a_{3} z^{-8},
$$

while the first sub-filter has an extra output for the notch component having the transfer function given by

$$
H_{n o t c h}(z)=0.5+b_{0} z^{-4} \text {. }
$$

The overall transfer function of the filter is given by

$$
\begin{aligned}
H_{T}(z) & =(1+G 0)\left(0.5+b_{0} z^{-4}\right) \\
& +G 1\left(0.5+a_{0} z^{-2}+a_{1} z^{-4}+a_{2} z^{-6}+a_{3} z^{-8}\right)^{P} .
\end{aligned}
$$

The parameter $P$ controls the order of the filter and has a value of 3 or 4 . The filter coefficients $b_{0}$ and $a_{0}$ through $a_{3}$ are pre-determined using only values that are powers of 2 for each desired center frequency. Therefore, they are easily hard-wired into the filter circuit using MUXs implementing simple shifting functions. The parameters $G 0$ and $G 1$ decide the gains of the notch and band-pass components, respectively. The parameters $G 0, G 1$, and $P$ are used to customize the frequency gain response of a filter configured for a given center frequency.

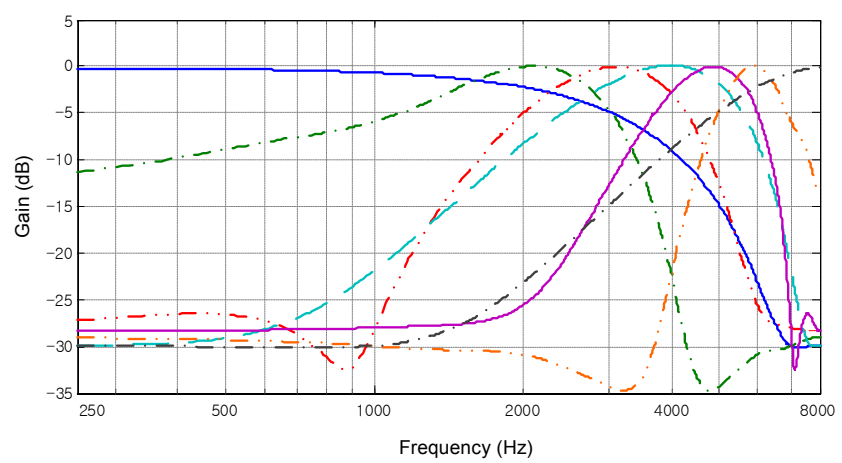

Figure 3. Typical frequency response of filters. (normalized)

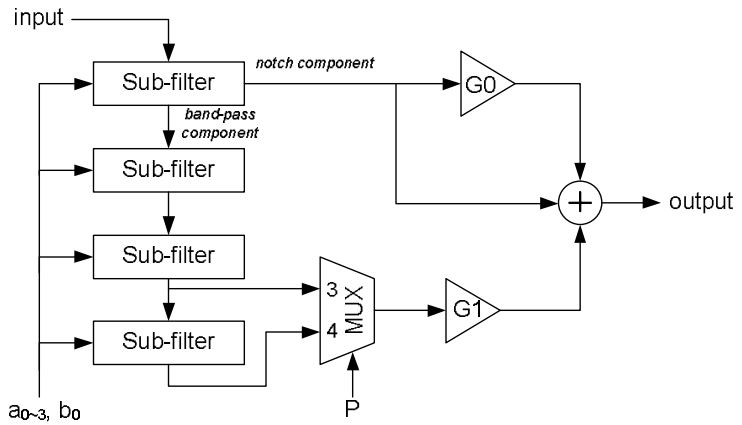

Figure 4. Finite impulse response filter. 


\section{Voice Activity Detector}

Voice activity detection (VAD) schemes have been mainly developed for use in communications and speech recognition applications [9]. Power dissipation is only a secondary consideration for these applications and as a result advanced VAD schemes employ computationally intensive algorithms such as the Fourier Transform to improve detection rates. Since such an implementation would be a large overhead for our design, a power efficient scheme is developed in this study.

Fig. 5 and Fig. 6 show the state diagram and block diagram of the proposed VAD circuit. Zero crossing rate (ZCR) and short-term energy are measured for each $32 \mathrm{~ms}$ timeframe. The justification for using zero crossing rate is the observation that speech signals tend to have less zero crossings during a set duration of time compared to random noise [9]. Short-term energy is measured by summing the square of the magnitude of the input samples during each time frame. The energy of the signal is assumed to be greater when voice is present. A sensitive soft threshold (220 for zero crossing rate and $10^{7}$ for short term energy in this study) and a more conservative hard threshold (160 for zero crossing rate and $2 \times 10^{7}$ for short-term energy in this study) are used to evaluate both measurements. When either the zero crossing rate or the short-term energy exceeds its hard threshold and the other measured value triggers its soft threshold, the VAD flag is set. Even when voice is no longer detected, there is a hangover period of $256 \mathrm{~ms}$ in which the VAD flag maintains its set status. The thresholds for each

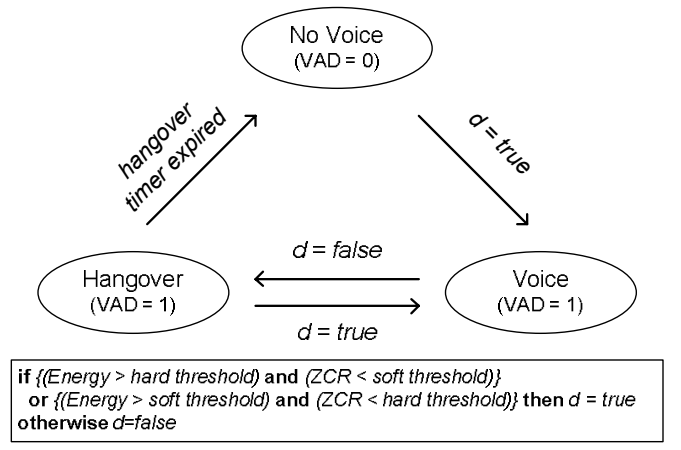

Figure 5. Voice activity detector state diagram.

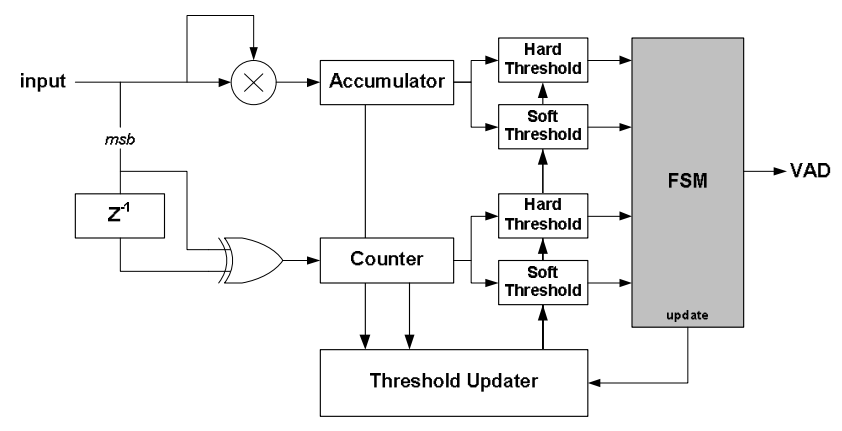

Figure 6. Proposed voice activity detector circuit. measurement are adaptively updated with respect to the averages of the measured values during prolonged periods of voice inactivity. Fig. 7 shows voice activity detection results for Korean speech in a laboratory environment with ambient noise. VAD remains set during brief pauses between words, thereby effectively reducing the number of mode transitions.

When the VAD flag is cleared and the DSP enters the silence mode, first of all, two of the three FIR filters comprising the filter bank are disabled by gating off the clock signal. At the same time, parameters for the remaining filter are corrected to compensate for the removal of two filters. The process is reversed when the VAD flag is restored.

\section{Fade-in/out Mixer}

The function of the mixer is to scale and combine the outputs from the filter bank to obtain the final full-band frequency gain compensation. The scaling is applied in powers of 2, allowing it to be implemented using barrel shifters instead of multipliers.

When transitioning between the active mode and the silence mode, a sudden inclusion or removal of signals in the output of the DSP may introduce glitches, which would cause discomfort to the user. In order to prevent this, a fading function is implemented in the mixer. As a result, the mixer is able to gradually fade-out signals when transitioning to the silence mode and fade-in those signals when returning back to the active mode. The fade-in/out effect is accomplished using up/down ripple counters to update the shift amount values of the scaling barrel shifters. The counters run on a lower clock than the rest of the DSP so that the maximum duration of the fade-in/out effect is $32 \mathrm{~ms}$.

\section{IMPLEMENTATION RESULTS}

All blocks of the DSP were synthesized using a $0.18 \mu \mathrm{m}$, $1.8 \mathrm{~V}$ CMOS mixed mode standard cell library. After placement and routing, transistor level simulations were carried out to address possible problems arising from operating at $0.9 \mathrm{~V}$. The DSP was implemented as part of a fully operational, fully integrated digital hearing aid chip [6].

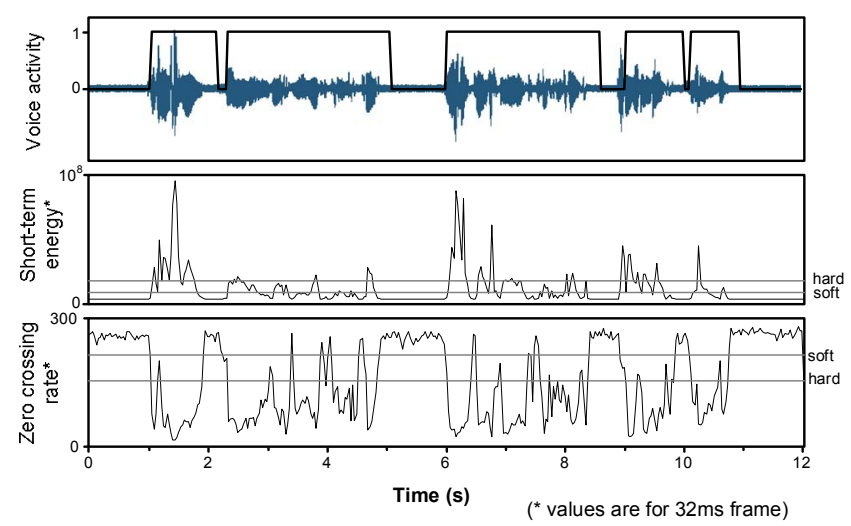

Figure 7. Voice activity detection results. 
A photo micrograph of the chip and DSP macro are shown in Fig. 8. The DSP integrates $40 \mathrm{k}$ gates in $0.5 \mathrm{~mm}^{2}$, and consumes $25 \mu \mathrm{W}$ in active mode and $9 \mu \mathrm{W}$ in silence mode while operating at $32 \mathrm{kHz}$. Under the generous assumption that the DSP operates in active mode $20 \%$ of the time it is powered on, a power reduction of $50 \%$ is achieved by using the proposed VAD scheme. The resulting $12 \mu \mathrm{W}$ average power consumption is on par with the previous work [2] while providing much higher flexibility.

The frequency gain compensation graph of the proposed DSP fitted against an audiogram of a typical case of hearing loss is shown in Fig. 9. This particular audiogram exhibits ski slope loss. The compensation is quite accurate to within $5 \mathrm{~dB}$ of the desired curve across the frequency spectrum. This is comparable to previous works that have a higher number of frequency bands and thus higher power consumption [4]. Fig. 10 shows the input and output signals of the DSP while transitioning from the silence mode to the active mode. No audible artifacts are observed during the transition thanks to the fade-in/out circuitry.

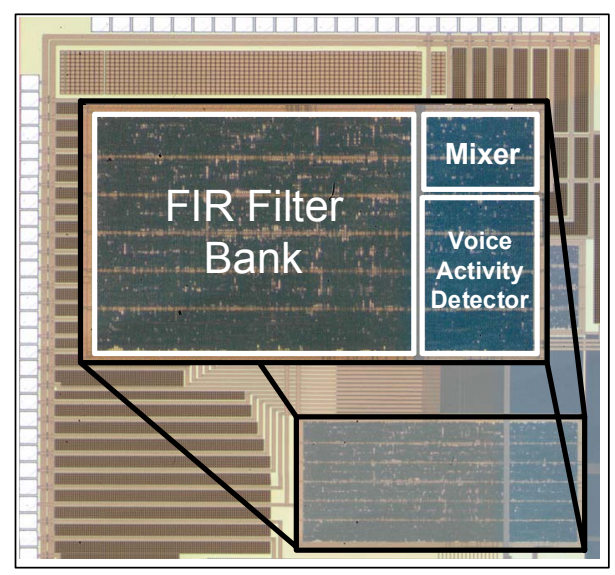

Figure 8. Photo of digital signal processor macro.

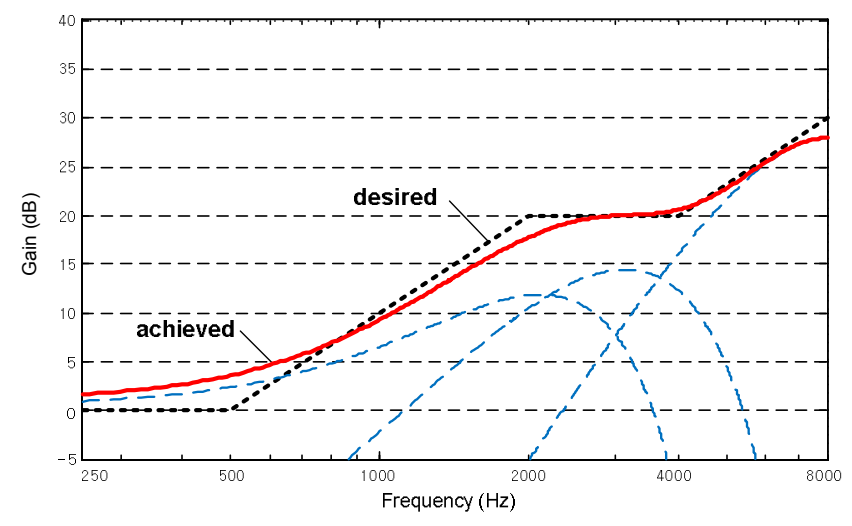

Figure 9. Frequency gain compensation result.
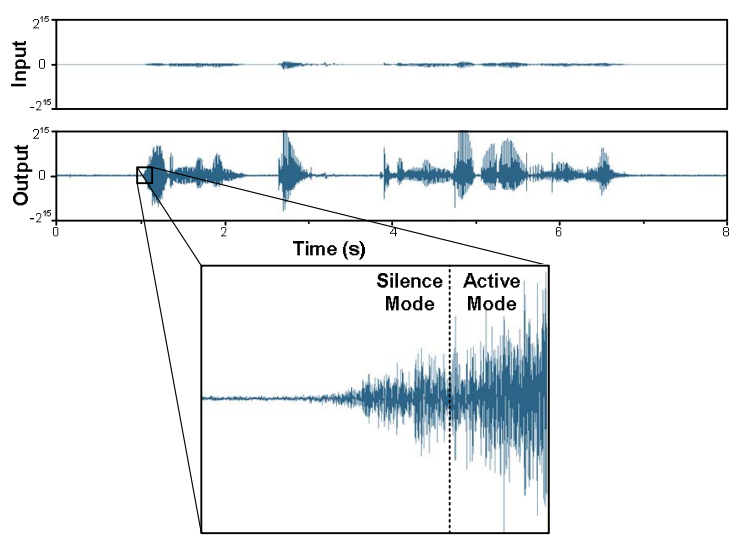

Figure 10. Input and output signal during mode transition.

\section{CONCLUSION}

A low power DSP with VAD based partial activation is implemented as part of a fully integrated hearing aid chip. It integrates three configurable hardware FIR filters to minimize power dissipation while obtaining high flexibility of seven channels. In addition, the computationally efficient VAD scheme allows for an additional maximum power saving of $60 \%$ when voice activity is not detected. The resulting power consumption is just $12 \mu \mathrm{W}$ when assuming a voice activity ratio of $20 \%$.

\section{REFERENCES}

[1] S. Kim, J.-Y. Lee, S.-J. Song, N. Cho, and H.-J. Yoo, "An energyefficient analog front-end circuit for a sub-1-V digital hearing aid chip," IEEE Journal of Solid-State Circuits, vol. 41, is. 4, pp. 876882, April 2006.

[2] J. Yoo, S. Kim, N. Cho, S.-J. Song, and H.-J. Yoo, “A 10-uW digital signal processor with adaptive-SNR monitoring for a sub-1V digital hearing aid," IEEE International Symposium on Circuits and Systems, May 2006.

[3] R. Brennan, and T. Schneider, "A flexible filterbank structure for extensive signal manipulations in digital hearing aids," IEEE Symposium on Circuits and Systems, vol. 6, pp. 569-572, May 1998.

[4] T. Lunner, and J. Hellgren, "A digital filterbank hearing aid-design, implementation and evaluation," International Conference on Acoustics, Speech, and Signal Processing, vol.5, pp. 3661-3664, 1991.

[5] P. Yanick and H. Drucker, "Signal processing to improve intelligibility in the presence of noise for persons with a ski-slope hearing impairment," IEEE Transactions on Acoustics, Speech, and Signal Processing, vol. 24, is. 6, pp. 507-512, Dec.1976.

[6] S. Kim, S.J. Lee, N. Cho, S.-J. Song, and H.-J. Yoo, "A fully integrated digital hearing aid chip with human factors considerations," to be presented at the IEEE International Solid-State Circuits Conference, Feb. 2007

[7] S. Kim, N. Cho, S.-J. Song, D. Kim, K. Kim., and H.-J. Yoo, “A 0.9V 96-uW digital hearing aid chip with heterogeneous sigma-delta DAC," IEEE Symposium on VLSI Circuits, pp. 55-56, June 2006

[8] “Hearing aid digital filter," U.S. Patent 6292571 B1, Sept. 18, 2001.

[9] K. El-Maleh, and P. Kabal, "Comparison of voice activity detection algorithms for wireless personal communications systems," IEEE Canadian Conference on Electrical and Computer Engineering, vol. 2, pp. 470-473, May 1997. 